\title{
A model for ultrasound modulated light in a turbid medium
}

Joseph L. Hollmann, Roarke Horstmeyer, Changhuei Yang, Charles A. DiMarzio

Joseph L. Hollmann, Roarke Horstmeyer, Changhuei Yang, Charles A. DiMarzio, "A model for ultrasound modulated light in a turbid medium," Proc. SPIE 8949, Three-Dimensional and Multidimensional Microscopy: Image Acquisition and Processing XXI, $89490 U$ (12 March 2014); doi:

10.1117/12.2041179

SPIE. Event: SPIE BiOS, 2014, San Francisco, California, United States 


\title{
A model for ultrasound modulated light in a turbid medium
}

\author{
Joseph L. Hollmann ${ }^{a, b}$, Roarke Horstmeyer ${ }^{c}$, Changhuei Yang ${ }^{c}$, and Charles A. DiMarzio ${ }^{a, b, d}$ \\ ${ }^{a}$ Electrical and Computer Engineering; Northeastern University, 440 Dana Research Center, \\ Boston, MA, USA; \\ ${ }^{b}$ Gordon Center for Subsurface Sensing and Imaging Systems; 360 Huntington Ave. Boston, \\ MA 02115, USA \\ ${ }^{c}$ Department of Electrical Engineering; California Institute of Technology, 1200 E California \\ Blvd, Pasadena, CA \\ ${ }^{d}$ Department of Mechanical and Industrial Engineering; Northeastern University, 440 Dana \\ Research Center, Boston, MA, USA;
}

\begin{abstract}
The ability to focus light in most tissue degrades quickly with depth due to high optical scattering. Researchers have investigated using both ultrasound (US) and light synergistically to overcome this difficulty.

Ultrasound has been utilized to modulated light within tissue to create a diffusive wave at that is modulated at the US frequency. Recently, there has been interest in the modulated sidebands which reside at optical frequency plus or minus the US frequency.

This paper will discuss a model for US-light interactions in a scattering medium. We will use this model to relate the radiance in the probe beam to the radiance in the diffusive wave. We will then employ the $\mathrm{P}-1$ approximation to the radiative transport equation to find the fluence and flux of the modulated wave. We will use these parameters to write a diffusion equation for the modulated wave that can be described in terms of the incoming optical power, and the US intensity and geometry.
\end{abstract}

\section{INTRODUCTION}

Current optical imaging techniques are inhibited by high scattering within tissue. Several optical methods, such as confocal microscopy, optical coherence tomography and diffuse optical tomography have attempted to circumvent this limitation but end up sacrificing resolution or depth of penetration, or both. However, ultrasound (US) does not suffer from the same problem and can be utilized for imaging at much greater depths than optical techniques. Ultrasound detects changes in density and is not sensitive to physiological parameters. Synergistically combining light and ultrasound accomplishes both.

One such technique utilizes an ultrasonically encoded wavefront for phase conjugation. ${ }^{1-3}$ The resulting signal may be used to create diffraction limited focal spots at millimeter depths within tissue. ${ }^{4}$ This paper will present a diffusion model for US-modulated light so the expected power may be calculated.

\section{ULTRASOUND MODULATION}

An US wave interacts with an optical signal in a turbid medium by modulating the field's optical pathlength. If the interaction is linear, this may be done by modulating the index of refraction due to condensing and rarification of the density of the medium. ${ }^{5}$ It may also be accomplished by inducing movement in the scatterers, though this is assumed to be minimal at high frequencies. ${ }^{2}$ For the purposes of this paper we will assume the primary interaction is due to index of refraction modulation and assume scatterer motion is negligible. The discussion that follows relies heavily on the work presented by Hollmann et al. ${ }^{6}$

Further author information: Send correspondence to Joseph Hollmann

: E-mail:joe1h@aol.com

Three-Dimensional and Multidimensional Microscopy: Image Acquisition and Processing XXI,

edited by Thomas G. Brown, Carol J. Cogswell, Tony Wilson, Proc. of SPIE Vol. 8949, 89490U

(C) 2014 SPIE · CCC code: 1605-7422/14/\$18 - doi: 10.1117/12.2041179

Proc. of SPIE Vol. $894989490 U-1$ 
We will analyze the field modulated by an infinitesimally small US dot to understand how the change in optical pathlength creates modulation. First, an optical wave may be described as,

$$
E=A \exp \left(j \omega_{o} t\right)
$$

where $A$ is the amplitude and $\phi$ is the phase of the field. If the field traverses an infinitesimally small US dot, it experiences a small delay or,

$$
E_{\text {mod }}=A \exp \left(j \omega_{o} t+\phi_{u s}\right)
$$

where the $\phi_{u s}$ is the phase delay due to the US dot. Assuming the phase delay is only due to a change in the index of refraction, the phase delay is given by.

$$
\phi_{u s}=M \exp \left(j \omega_{u s} t\right)+M^{*} \exp \left(-j \omega_{u s} t\right),
$$

where $\angle M$ is the phase delay relative to the US oscillation and $|M|$ is the maximum induced phase delay. The maximum phase delay introduced by the US dot is given as,

$$
|M|=k \Delta n d s
$$

where $k=2 \pi / \lambda_{o}$ is the magnitude of the wave vector in vacuum, $\Delta n$ is induced change in index of refraction, and $d s$ is the path across the US dot. As shown elsewhere, ${ }^{6}$ the resulting field modulated into the positive sideband $\left(f_{o}+f_{u s}\right)$ is given by,

$$
E_{+}=j M A \exp \left(j \omega_{o} t\right) M e^{j \omega_{u s} t},
$$

where $j$ represents a 90 degree phase shift between the incident probe beam and the modulated sideband. The total modulated field at a point may be found by integrating over the angularly resolved field, $F(\hat{\Omega})$, at a point or,

$$
E_{+}=\iint F_{+}(\hat{\Omega}) d^{2} \hat{\Omega}
$$

where $d^{2} \hat{\Omega}$ is an infinitesimally small solid angle. The angularly resolved modulated field, $F_{+}(\hat{\Omega})$ results in a modulated radiance given by,

$$
L_{+}(\vec{r}, \hat{\Omega}, t)=\frac{1}{\eta}\left|F_{+}(\hat{\Omega})\right|^{2},
$$

where $\eta$ is the impedance of the medium. We see that the radiance modulated into the positive sideband is not linearly related to the modulation. However, the radiance modulated at the US frequency is because it is the result of the angularly resolved probe and modulated field mixing or,

$$
L_{+}(\vec{r}, \hat{\Omega}, t)=\frac{1}{\eta}\left|F_{+}(\hat{\Omega}) F_{0}^{*}\right|
$$

where $F_{0}$ is the angularly resolved unmodulated field.

We will utilize this relationship later to develop a Green's function for the modulated diffusive wave with a frequency of $f_{u s}$. In a diffusive medium, the field and resulting radiance may be found by rigorously solving Maxwell's equations using a finite-difference time-domain (FDTD) simulation. ${ }^{2}$ However, these simulations are computationally intensive and typically may only be solved for simulation spaces that are smaller than a millimeter. In the next section, we will relate these parameters to the diffusive radiance. This will allow us to develop a Green's function for the modulated diffusive wave that may be solved for much larger media with minimal computational effort.

\subsection{Diffusion}

The radiative transport equation (RTE) describes the optical radiance distribution in a turbid medium. The RTE may be analytically solved for a limited number of idealized geometries. However, the RTE may be simplified by expanding the radiance in terms of its spherical harmonics and truncating the expansion after the second 
term. ${ }^{7,8}$ The result is that the radiance may be described as follows,

$$
L(\vec{r}, \hat{\Omega}, t)=\frac{1}{4 \pi} \Phi(\vec{r}, t)+\frac{3}{4 \pi} \vec{J}(\vec{r} . t) \cdot \hat{n}+\ldots,
$$

where $\Phi$ is the fluence rate and represents an omni-directional irradiance term, or

$$
\phi(\vec{r}, t)=\iint L(\vec{r}, \hat{\Omega}, t) d^{2} \hat{\Omega} .
$$

The flux or directionality of the power flow is given by $\vec{J}$, or

$$
J(\overrightarrow{\vec{r}}, t)=\iint L(\vec{r}, \hat{\Omega}, t) \cdot \hat{\Omega} d^{2} \hat{\omega}
$$

where $\hat{n}$ is a unit vector parallel to the energy flow. However, this approximation eliminates most of the directionality in the medium. To help satisfy this approximation, $\mu_{s}$ is replaced with a reduced scattering coefficient, $\mu_{s}^{\prime}=\mu_{s}(1-g)$ and the medium is approximated as isotropically scattering. This is known as the diffusion or $P_{1}$ approximation and all higher order terms (represented by the dots in Equation 9) are dropped.

Fick's law provides the relationship between the gradient of the fluence rate and the flux or,

$$
\vec{J}(\vec{r}, t)=-\frac{D}{c} \nabla \Phi(\vec{r}, t),
$$

where $D=c /\left(3\left(\mu_{s}^{\prime}+\mu_{a}\right)\right)$ is the diffusion coefficient. This coefficient is related to the transport mean-free path by $\ell_{t}^{\prime}=3 D / c$.

The result of this approximation may be seen in the cartoon in Figure 1. A turbid medium is interrogated by an optical source that emits light along the depth direction in Figure 1a. The highly directional optical source is replaced with an isotropic source that is located $1 \ell_{t}^{\prime}$ within a new medium as shown in Figure 1b. This new medium now contains isotropic scatterers and is described by its reduced scattering coefficient. It is clear to see from these cartoons that the diffusion approximation is not valid where the optical field is highly directional. Typically, it is assume the radiance distribution is valid at distances greater than $1 \ell_{t}^{\prime}$ from the source.

The turbid medium-air boundary is accounted for utilizing extrapolated boundary conditions. ${ }^{8}$ This stipulates a point source with an equivalent but negative magnitude is located above the turbid medium. The negative source's job is similar to account for the optical power exiting the medium and has a $\mathrm{z}^{-}$-axis position given by,

$$
z_{b}=-\ell_{t}^{\prime}-2 A D
$$

where $A$ is a constant that depends on the index of refraction mismatch between the turbid and ambient media. Tables for this constant for different indicies are given elsewhere. ${ }^{9,10}$ However, we will assume the indicies are matched and $A=1$.

The fluence rate resulting from the superposition of point sources may be utilized to calculate the diffuse reflectance or,

$$
R(\vec{r}, t)=\frac{1}{4} \Phi(\vec{r}, t)+\frac{1}{2} \vec{J}(\vec{r}, t) \cdot \hat{n} .
$$

The fluence rate is primarily changing along the $\mathrm{z}^{-}$-axis so we approximate the second part of the equation as,

$$
\vec{J} \cdot \hat{n} \approx-\frac{D}{c} \frac{\delta}{\delta z} \Phi
$$

where we utilized Fick's law to relate the fluence rate to the flux.

\subsection{Modulated Diffusive Wave}

This section will discuss light modulated into the diffusive wave. Light is focused to a point within tissue and is modulated by a focused US wave as shown in Figure 2a. For the purposes of this discussion, we will approximate 


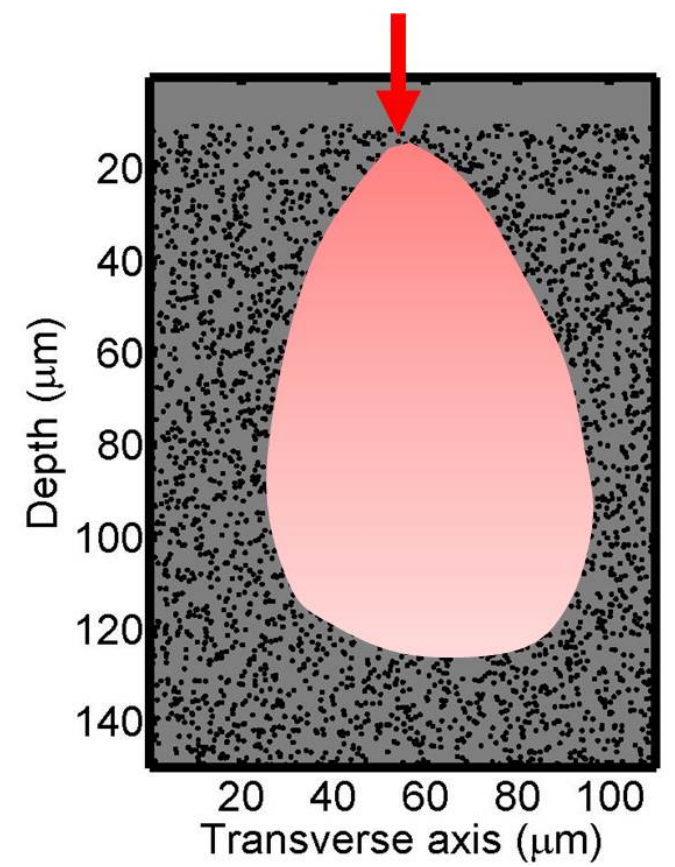

(a)

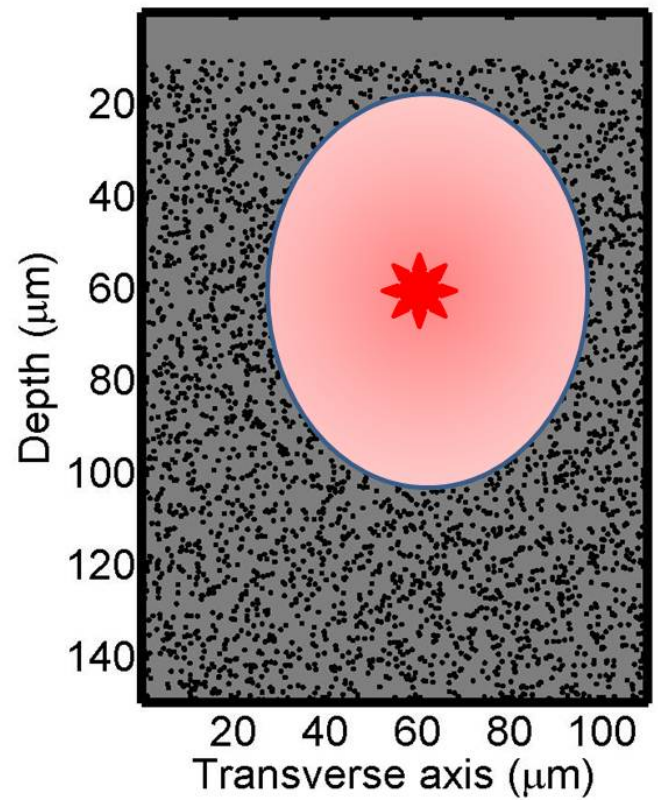

(b)

Figure 1. a) An infinitesimally narrow optical source (red) is normally incident upon a scattering medium (gray). b) The optical distribution may be described as coming from an equivalent point source (red star).

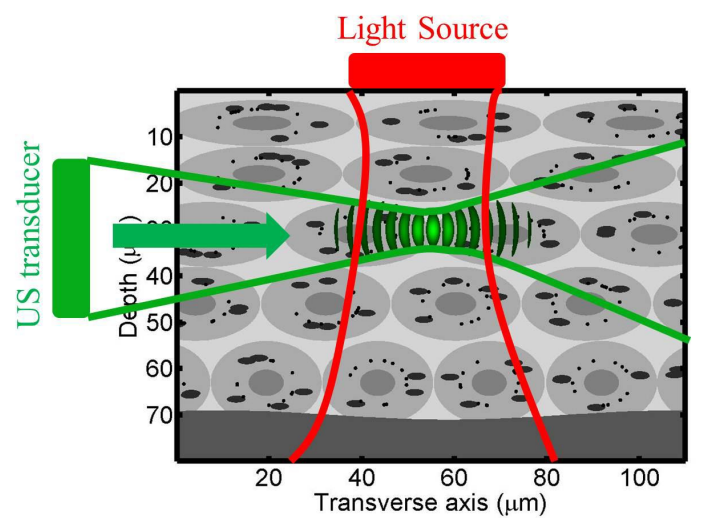

(a)

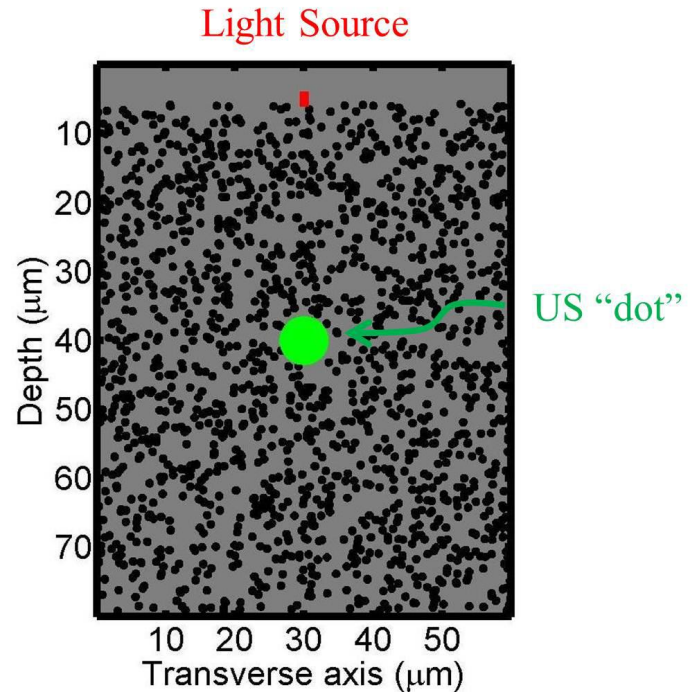

(b)

Figure 2. (a) A Gaussian ultrasound wave (green) focused in a turbid medium illuminated by a focused optical source. (b) The US beam is replaced with a sphere (green) and the optical source is replaced with an infinitesimally narrow photon beam.

the US dot as a sphere and the focused optical source is replaced by an infinitesimally narrow photon beam as shown in Figure 2b. The tissue will be modeled as a homogeneous turbid medium with uniform scattering and absorption properties. These approximations will allow for common solutions to the diffusion approximation to be utilized.

The incident light, or probe beam, may now be modeled using the diffusion approximation in Equation 9 . The incident optical source is replaced with an equivalent point source that is located one transport mean-free 


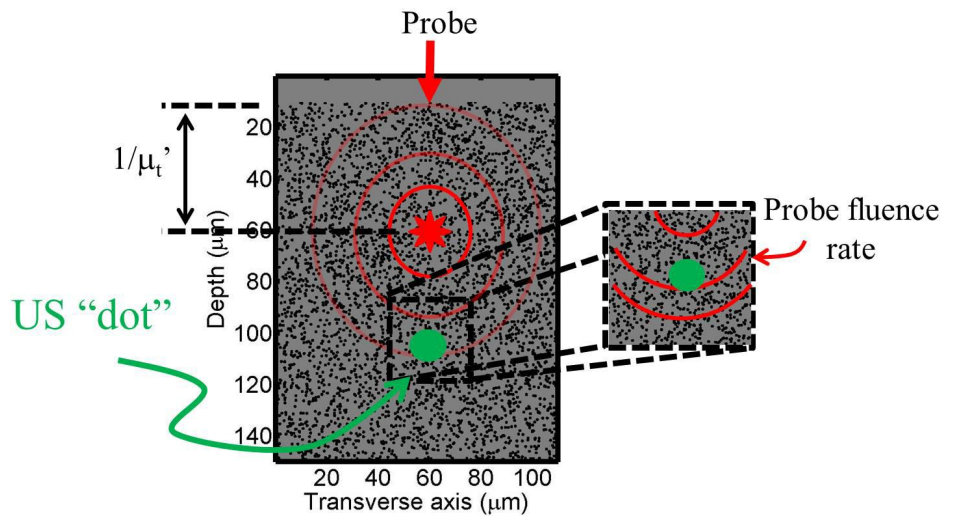

Figure 3. The incident probe beam is approximated by an equivalent isotropic source (red star) located 1 transport meanfree path within the medium. The diffusion equation may be utilized to solve for the fluence rate and flux of the probe beam at the US sphere (green).

path within the medium, as illustrated by the red star in Figure 3. The diffusion equation can be utilized to approximate the radiance within the medium for distances greater than one $\ell_{t}^{\prime}$ away from the source.

As mentioned above, the radiance modulated at the US frequency is linearly related to the US modulation. The reader is reminded that the US sphere modulates the index of refraction through rarification and compression of the medium. This, in turn, modulates the optical pathlength of light traversing the sphere. The result is the field is phase modulated. This allows us to relate the probe at a specific location in the US beam to the modulated radiance or,

$$
L_{+}(\vec{r}, \hat{\Omega})=L_{0}(\vec{r}, \hat{\Omega}) \Delta n k,
$$

where we utilized the definition of the magnitude of the US modulation given in Equation 4. The modulated radiance may then be utilized to build a diffusion approximation for the modulated wave. We now utilize the relationship between the radiance and fluence rate to obtain,

$$
\Phi\left(f_{u s}\right)=2 j \Phi_{0} \Delta n k .
$$

where $\Phi_{0}$ is the probe fluence rate. Similarly, the modulated flux is given by,

$$
\vec{J}\left(f_{u s}\right) d s=2 j \overrightarrow{J_{0}} \Delta n k d s,
$$

where $\vec{J}_{0}$ is the probe flux.

\subsection{Modulated Source}

As mentioned above, the diffusion equation utilizes an equivalent point source to describe the probe radiance within the medium. The same may be done for the modulated diffusive wave modulated by an US sphere with a frequency, $f_{u s}$, at a depth, $z_{u s}$. The equivalent isotropic source (shown in Figure 4a) has some power and an offset. Figure $4 \mathrm{~b}$ illustrates the resulting isotropic point source's location within the medium. Their is also a negative point source modulated at the same frequency located above the turbid medium to account for the extrapolated boundary conditions.

\subsubsection{Modulated Power}

The modulated power may be found by integrating the modulated radiance (from Equation 16) over all solid angles and the volume of the US sphere, or

$$
P\left(f_{u s}\right)=\iiint_{V} \iint_{\hat{\Omega}} L_{u s}(\vec{r}, \hat{\Omega}) d^{2} \hat{\Omega} d^{3} V,
$$




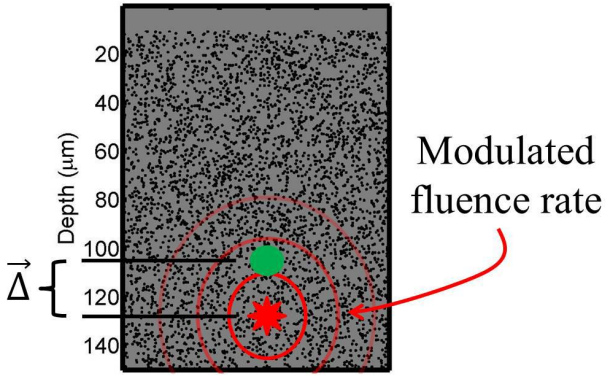

(a)

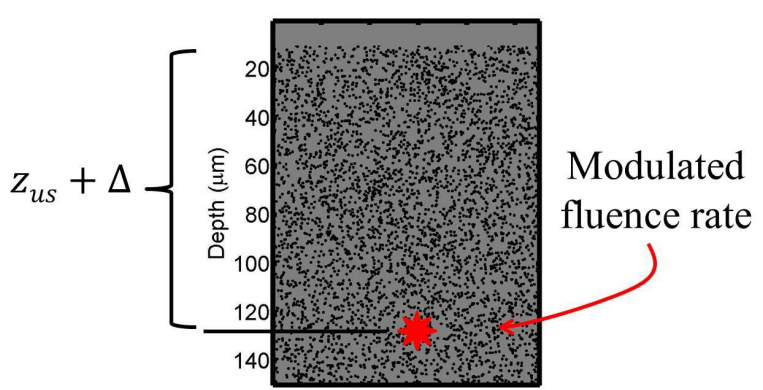

(b)

Figure 4. (a) The US sphere (green) and the equivalent point source for the modulated diffusive wave (red star). The modulated point source is offset from the US dot. (b) The equivalent source's new depth location.

where $V$ is the volume of the sphere. Though complicated, Equation 10 illustrates the interior integrations result in the fluence rate. The result is that the power modulated into the sideband may be written as,

$$
P\left(f_{u s}\right)=\iiint_{V} \Phi_{u s} d^{3} V=j 2 \iiint_{v} \Phi_{0} \Delta n k d^{3} V,
$$

where we utilized Equation 17 to relate the power to the probe fluence rate.

\subsubsection{Offset}

The probe diffusive wave has a flux at the US sphere that is modulated into the diffusive wave as well. This may be accounted for by creating an offset for the modulated equivalent isotropic source in the direction of the flux. Hollmann et al. ${ }^{6}$ showed this offset may be found by,

$$
\vec{\Delta}=\frac{\overrightarrow{J_{0}}}{\Phi_{0}} \ell_{t}^{\prime}
$$

We see the offset direction depends on the direction of the flux. The reader is reminded the flux flows away from the equivalent point source we stipulated earlier. This suggests the modulated centroid will be located at a depth greater than the US modulation depth if the modulation occurs greater than one transport mean-free path in the medium.

\subsubsection{Boundary Conditions}

The boundary between the turbid and ambient media must be accounted for as well. We accomplish this by introducing another negative point source, modulated at the US frequency, with a position above the $\mathrm{z}-$ axis given by,

$$
z_{c}=-z_{u s}-\Delta z-2 A D
$$

The index of refraction has not changed, so $A=1$.

\section{RESULTS}

A $633 \mathrm{~nm}$ probe beam is normally incident upon a turbid medium and is modulated by an ultrasound sphere with a frequency $f_{u s}=100 \mathrm{MHz}$. The sphere has a radius of approximately $15 \mu \mathrm{m}$ and modulates local index of refraction with $\Delta n=0.001$. The medium a reduced scattering coefficient $\left(\mu_{s}^{\prime}=10 \mathrm{~cm}^{-1}\right)$ and an absorption coefficient $\left(\mu_{a}=0.1 \mathrm{~cm}^{-1}\right)$. This results in a mean-free path of approximately $1 \mathrm{~mm}$.

The US sphere is moved to discrete locations along the $\mathrm{z}^{-}$-axis and the diffusion approximation is utilized to solve for the fluence rate and flux of the probe beam. The US sphere modulates the probe and the resulting 


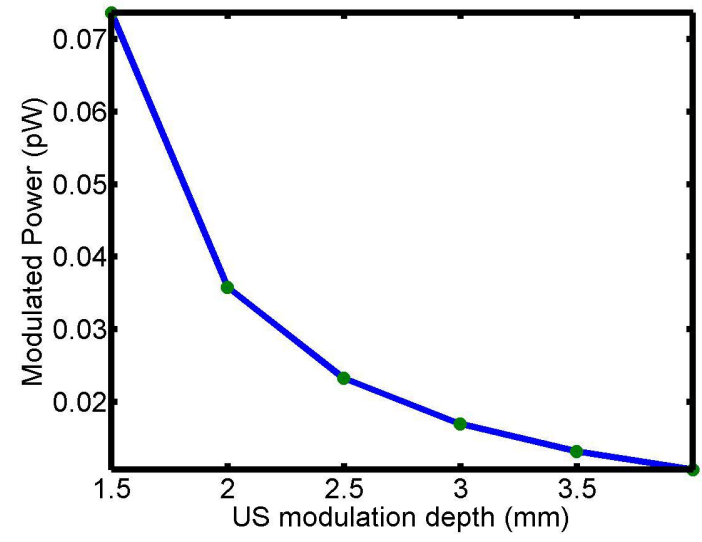

(a)

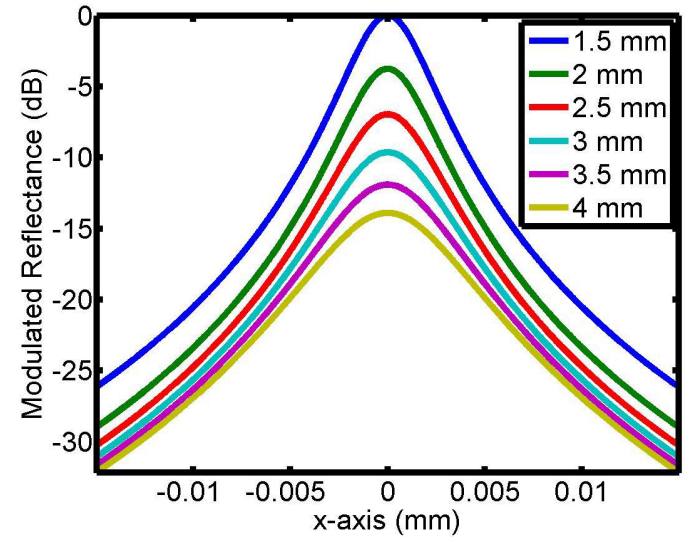

(b)

Figure 5. (a) The optical power modulated into the diffusive wave at $f_{u s}$ as the ultrasound sphere is moved deeper into the turbid medium. (b) The resulting diffuse reflectance for the US modulated light in decibels.

diffusive wave has a frequency of $f_{\text {us }}$, a power given by Equation 2.3.2, and an offset from the US sphere given by Equation 21. The US sphere is located at discrete depths ranging from $1.5 \mathrm{~mm}$ to 4 mm or approximately one-and-a-half to four $\ell_{t}^{\prime}$. The resulting modulated power is illustrated in Figure 5a. The green circles indicate the depth location of the US dot. As expected, we see the power exponentially decays as the US sphere is moved deeper in the medium.

The resulting modulated reflection is illustrated in Figure $5 \mathrm{~b}$ in decibels. Each curve is the result of the US dot at different depths. As expected, we see the peak of the modulated light decreases with depth. However, it is also interesting to note the slope of the modulated light also varies with depth.

\section{CONCLUSIONS}

This paper explained a diffusion model for diffusive light modulated by an ultrasound beam. We first approximate the turbid medium as having homogeneous scattering and absorption properties and utilize the diffusion approximation to solve for the radiance distribution of the probe beam. The US wave is approximated as an US sphere and calculate the probe's radiance at the US beam. This is utilized to compute the power and position of a modulated equivalent point source. This new point source is utilized to find the diffuse modulated reflectance. These relationships were utilized to find the modulated diffusely reflected light due to an ultrasound sphere.

\section{REFERENCES}

1. X. Xu, H. Liu, and L. Wang, "Time-reversed ultrasonically encoded optical focusing into scattering media," Nature Photonics, 2011.

2. J. L. Hollmann, R. Horstmeyer, C. Yang, and C. A. DiMarzio, "Analysis and modeling of an ultrasoundmodulated guide star to increase the depth of focusing in a turbid medium," Journal of Biomedical Optics 18(2), pp. 025004-025004, 2013.

3. C. A. DiMarzio, Optics for Engineers, Crc Press, 2011.

4. Y. M. Wang, B. Judkewitz, C. A. DiMarzio, and C. Yang, "Deep-tissue focal fluorescence imaging with digitally time-reversed ultrasound-encoded light," Nature Communications 3, June 2012.

5. C. A. DiMarzio and T. W. Murray, "Medical imaging techniques combining light and ultrasound," Subsurface Sensing Technologies and Applications 4, pp. 289-310, October 2003.

6. J. L. Hollmann, R. Horstmeyer, C. Yang, and C. A. DiMarzio, "Diffusion model for ultrasound-modulated light," Journal of Biomedical Optics (accepted).

7. D. Boas, Diffuse photon probes of structural and dynamical properties of turbid media: theory and biomedical applications. PhD thesis, University of Pennsylvania, 1996. 
8. R. C. Haskell, L. O. Svaasand, T.-T. Tsay, T.-C. Feng, M. S. McAdams, and B. J. Tromberg, "Boundary conditions for the diffusion equation in radiative transfer," J. Opt. Soc. Am. A 11, pp. 2727-2741, Oct 1994.

9. S. E. Orchard, "Reflection and transmission of light by diffusing suspensions," J. Opt. Soc. Am. 59, pp. 15841597, Dec 1969.

10. J. L. Hollmann, "Multi-layer diffusion approximation for photon transport in biological tissue," 2008. 